\title{
Multi-Targeted Approach to Treat Drug Resistant CML Using Natural Compounds: A Double Edged Sword
}

\author{
Phani Krishna P*1,2, Kalubai Vari Khajapeer ${ }^{1}$, Anasooya P Balakrishnan ${ }^{1}$ and Baskaran Rajasekaran ${ }^{1,2}$ \\ ${ }^{1}$ Department of Biochemistry and Molecular Biology, School of Life Sciences, Pondicherry University, India \\ ${ }^{2}$ DBT-Interdisciplinary program in Life sciences, School of Life sciences, Pondicherry University, India
}

*Corresponding author: Phani Krishna P, PhD Scholar, Department of Biochemistry and Molecular Biology \& DBT-Interdisciplinary program in Life sciences, School of Life sciences, Pondicherry University, India

Submission: March 03, 2018; Published: July 16, 2018

\begin{abstract}
Chronic myelogenous leukemia (CML) is a hematopoietic stem cell disorder which arises due to translocation of ABL gene on chromosome 9 to BCR gene present on chromosome 22. This produces BCR-ABL oncogene which is the main cause of CML. Imatinib mesylate has been the choice for the treatment of initial chronic phase of the disease. However, through BCR-ABL gene over expression, kinase domain mutations, importantly T315I CML cells acquire resistance to imatinib and other second-generation tyrosine kinase inhibitors. In this mini-review, we will be analyzing the normal functions of c-Abl and the aberrant signaling pathways activated upon its loss of regulation. The causes of drug resistance and some of the possible routes to combat drug resistance is defined. The natural compounds and their significance in drug discovery and the efficacy of tyrosine kinase inhibitors by combinational treatment with natural compounds to overcome the adverse side effects and drug resistance also examined.
\end{abstract}

Keywords: Chronic myelogenous leukemia (CML); BCR-ABL; Imatinib; Drug resistance; Natural compounds; Tyrosine kinase inhibitors

\section{Mini Review}

Chronic myelogenous leukemia (CML) is characterized by excessive proliferation of myeloid lineage of cells. The abnormality is caused due to constitutive tyrosine kinase activity of fusion protein $\mathrm{Bcr}-\mathrm{Abl}$, resulting from reciprocal chromosomal translocations of ABL from chromosome 9 to BCR on chromosome 22 [1]. The normal cellular $\mathrm{Abl}$ (c-Abl) is a multi-domain protein that belongs to non-receptor tyrosine kinase family. It phosphorylates proteins at tyrosine residues. Phosphorylation activity of Abl kinase is under allosteric regulation by its $\mathrm{SH} 2$ domain $[2,3]$. Abl influences several protein-protein interactions and enzymatic activity and localization. C-Abl associates a variety of sub cellular proteins including signaling adaptors, kinases, phosphatases, cell cycle regulators, transcription factors and cytoskeletal proteins [2,4]. DNA damage response of c-Abl is mediated by Ataxia Telangiectasia Mutated (ATM) and Abl critically modulates the epigenetic and non-epigenetic regulators of DNA damage and apoptosis [5,6]. Normal c-Abl is auto-regulated both by inter and intra-molecular interactions of its $\mathrm{N}$ and $\mathrm{C}$ terminal domains [7]. The Bcr-Abl fusion protein, produced as a result of the translocation posses consistently elevated tyrosine kinase activity [8]. Normal cellular Abl p145 shuttles between nuclear and cytoplasm compartments. The fusion protein Bcr-Abl retains in the cytoplasm and activates aberrant cell signaling pathways that leads to the halt of apoptosis and induces proliferation [9]. Furthermore, Bcr-Abl protein directly or indirectly activates of Signal transducer and activator transcription 5 (STAT5)/B-cell lymphoma-extralarge (Bcl-xL), extracellular signal-regulated kinase 1/2 (Erk-1/2), phosphatidylinositide 3-kinase (PI3K)/Ak strain transforming (Akt), Src signaling molecules by Phosphorylation $[9,10]$. The auto-phosphorylated Bcr-Abl provides the docking site, tyr-177 of Bcr domain, for these signaling molecules [11]. Constitutively active Bcr-Abl further drives several survival pathways that provide proliferative advantage and drug resistance in CML [12]. Src kinases, such as Lyn, Hck, Fgr, gets activated downstream of Bcr-Abl signaling upon direct complex formation with the Bcr-Abl and also involved in the activation of other downstream signaling molecules thus Bcr-Abl requires Src kinases for its transforming activity [13]. Therefore, simultaneously targeting Bcr-Abl and Src kinases is proven an effective strategy by Dasatinib, a dual tyrosine kinase inhibitor [14]. Inhibition of Src family kinase (SFK) with pyrrolo pyrimidine inhibitor, A-419259, inhibits the cell growth and promote apoptosis in CML cell lines which indicates their transforming potential in CML progression [15]. The transition of chronic phase to accelerated blast phase in CML progression requires Src kinases Lyn, Hck, Fgr. Lyn plays a role in cytokine 
signaling in a variety of cells and shown to have role in cell growth and apoptosis in hematopoietic cells $[16,17]$. Lyn over expression was observed in imatinib resistant CML cells it has inhibitory effect on apoptosis and can provide the resistance to the Imatinib and Nilotinib [18]. Both the Src kinases Lyn and Hck get activated upon complex formation with the Bcr-Abl by kinase independent mechanism. Src kinases phosphorylates tyr177 residue of Abl kinase thus substituting the role of auto-Phosphorylation activity of kinase defective Bcr-Abl [19]. Src kinases play different roles in different lineage of hematopoietic cells in Bcr-Abl dependant and independent mechanisms and provide survival advantage by the activation of different downstream targets [20].

High throughput screening (HTS) and combinatorial chemistry in the 90's, leads the anti-cancer drug discovery towards targeted therapies [21]. Gleevec and Herceptin are the successful out comes from the targeted therapies [22,23]. In the case of CML, selective inhibition of Abl kinase is an attractive strategy in the CML treatment. Targeted inhibition of deregulated Abl kinases enzymatic activity using ATP binding site inhibitors, interfere with Abl substrate Phosphorylation and thereby inhibit tumor cell proliferation, progression and survival. Highly selective imatinib is ineffective upon mutation which brings the conformational flexibility to the Bcr-Abl. T315I point mutation is one that brings conformational change at the ATP binding pocket of Bcr-Abl there by resistance to the imatinib. The acquired resistance for one targeted drug could be compensated with an alternative drug or by simultaneously targeting another essential protein involved in the pathogenesis process [24]. In silico screening of drug like molecules for the Bcr-Abl wild type and T315I mutant results in identification of some of the novel leads to combat drug resistance [25].

Currently, there is a need to identify novel tyrosine kinase inhibitors that target the different region of Bcr-Abl and the other downstream molecules of Bcr-Abl activated pathways. In addition, new ways of TKI utilization must be investigated in order to overcome adverse side effects involved with treatment and to improve patient survival.

Traditional Chinese Medicine (TCM) is used as alternative or complementary medicine and has been used for years to treat different cancers. TCM components stabilize tumor lesions, improve symptoms, enhances the quality of life, prolong survival time [21]. Natural compounds of TCM are highly diverse and chemically complex and the rich source for the identification of novel molecules for different cellular targets. Natural inhibitors identified from ZINC database by In Silico virtual screening against Bcr-Abl were shown to be promising upon in vitro testing [26]. Natural compounds possess privileged structures and are chemically complex and diverse that interacts with several cellular targets simultaneously to bring the optimal outcome. Using natural compounds as drugs has been proven as safe and exhibits less side effects [27].

Current tyrosine kinase inhibitors imatinib, Nilotinib, Dasatinib, Bosutinib and Ponatinib are potent in Bcr-Abl targeted treatment of CML but the side-effects and drug-resistant mutations in BCR-
ABL limit their capability. Natural compounds that inhibit Bcr-Abl or other downstream targets involved in progression of CML need to be identified as an alternative or as a complementary to these synthetic drugs. Some reports showed combination of natural compounds with synthetic drugs minimizes the dosage toxicity of the drug and optimizes the response compared to single agent alone [28]. The drug combination of current synthetic tyrosine kinase inhibitor ponatinib and natural compounds produced in the roots of the plant Coleus forskohlii showed effectiveness towards highly drug resistant mutant T315I along with other CML cell lines [29]. A triterpenoid Stelletin B isolated from marine sponge Jaspisstellifera were shown to be effective as a single agent or in combination with imatinib on CML cell lines. It mediates apoptosis in CML cells through the inhibition of STAT-5, PI3K and also it modulates the drug efflux mechanism of multidrug resistance proteins MDR1 and MRP1 in CML cells [30]. Similarly $\alpha$-bisabolol, a small plant-derived oily sesquiterpene alcohol also revealed striking synergism with imatinib and nilotinib on Bcr-Abl positive cell lines. It illustrated the pro-apoptotic effect by Bcr-Abl independent cell death mechanism caused by the loss of plasma and mitochondrial membrane integrity [31]. Homoharringtonine is another natural alkaloid produced from various Cephalotaxus species. In CML its effectiveness is independent of Bcr-Abl mutations. It showed to have potency over the T315I mutant Bcr-Abl [32].

Cancer cells exhibit abnormal ROS level with high oxidative stress. Some natural compounds further promote the oxidative stress by the generation of ROS and induce oxidative stress-mediated cell death. Pharmacological ROS modulation perspective could be utilized for selective killing of cancer cells [33]. Natural compound $\beta$-phenyl ethyl isothiocyanate (PEITC) sensitizes CML cells and induce cell death by the generation of ROS. It demonstrated to have effectiveness in killing Gleevec resistant cells harboring T315I mutation [34]. The fungal metabolite chaetocin inhibits thioredoxin reductase- 1 thereby induces oxidative stress and showed potency in the eradication of leukemic stem cells (LSCs) which are resistant to the drug imatinib. The enhanced efficacy of chaetocin observed in the presence of bone marrow stromal cytokines and growth factors (BMSFs) [35]. Hydroxychavicol ( $\mathrm{HCH}$ ) a component present in the alcoholic extracts of Piper betel leaves induces the apoptosis in CML cells including drug-resistant T315I cells. It mediates apoptosis by ROS dependent JNK activation [36]. The tyrosine Phosphorylation activity of Bcr-Abl increased upon treatment with ROS generating H2O2 [37] but interestingly the natural compounds that generate ROS inhibit the growth of Bcr-Abl positive cells by Bcr-Abl independent mechanism. It indicates natural compounds potential to modulate ROS mediated signaling towards the desired therapeutic effect. The mechanism by which they mediate the therapeutic effect requires thorough investigation.

\section{Conclusion}

The clear understanding of disease pathology and involvement of different downstream singling molecules could facilitate the design of new treatments for CML. Natural compounds could interact with multiple targets simultaneously and bring the 
potential outcome. In addition, natural compounds can be used as an adjunct with TKI inhibitors, providing one of the best approaches to treat drug-resistant CML.

\section{Acknowledgment}

This article is dedicated in loving memory to our guide and Associate Professor Dr. R. Baskaran, who met with an un-expected demise on June 2, 2018. He was a vibrant professor, well-wisher, wise and an impartial man. We are grateful to him forever.May his soul rest in peace.

\section{References}

1. Newel PC, Hungerford DA (1960) A minute chromosome in human chronic granulocytic leukemia. Science 132: 1497.

2. Colicelli J (2010) ABL tyrosine kinases: evolution of function, regulation, and specificity. Sci Signal 14(3): 1-46.

3. Lamontanara AJ, Georgeon S, Tria G, Svergun DI, Hantschel O (2014) The SH2 domain of Abl kinases regulates kinase autophosphorylation by controlling activation loop accessibility. Nature Communications 5 : $5470-6470$.

4. Ardito F, Giuliani M, Perrone D, Troiano G, Lo Muzio L (2017) The crucia role of protein phosphorylation in cell signaling and its use as targeted therapy. International Journal of Molecular Medicine 40(2): 271-80.

5. Mahajan K, Mahajan NP (2015) Cross talk of tyrosine kinases with the DNA damage signaling pathways. Nucleic Acids Res 43(22): 1058810601.

6. Ow SH, Chua PJ, Bay BH (2017) Epigenetic regulation of peroxiredoxins: Implications in the pathogenesis of cancer. Experimental Biology and Medicine 242(2): 140-147.

7. Nagar B, Hantschel O, Young MA, Scheffzek K, Veach D, et al. (2003). Structural basis for the autoinhibition of c-Abl tyrosine kinase. Cell 112(6): 859-871

8. Deininger MW, Vieira S, Mendiola R, Schultheis B, Goldman, et al. (2000) BCR-ABL tyrosine kinase activity regulates the expression of multiple genes implicated in the pathogenesis of chronic myeloid leukemia. Cancer Research 60(7): 2049-2055.

9. Steelman LS, Pohnert SC, Shelton JG, Franklin RA, Bertrand FE, et al (2004) JAK/STAT, Raf/MEK/ERK, PI3K/Akt and BCR-ABL in cell cycle progression and leukemogenesis. Leukemia 18(2): 189-218.

10. Deininger MW, Goldman JM, Melo JV (2000) The molecular biology of chronic myeloid leukemia. Blood 96(10): 3343-3356.

11. He Y, Wertheim JA, Xu L, Miller JP, Karnell FG, et al. (2002) The coiledcoil domain and Tyr177 of bcr are required to induce a murine chronic myelogenous leukemia-like disease by bcr/abl. Blood 99(8): 2957-2968.

12. Danisz K, Blasiak J (2013) Role of anti-apoptotic pathways activated by $\mathrm{BCR} / \mathrm{ABL}$ in the resistance of chronic myeloid leukemia cells to tyrosine kinase inhibitors. Acta Biochimica Polonica 60(4): 503-14.

13. Danhauser RS, Warmuth M, Druker BJ, Emmerich B, Hallek M (1996) Activation of Src kinases p53/56lyn and p59hck by p210bcr/abl in myeloid cells. Cancer Research 56(15): 3589-3596.

14. Hu Y, Swerdlow S, Duffy TM, Weinmann R, Lee FY, et al. (2006) Targeting multiple kinase pathways in leukemic progenitors and stem cells is essential for improved treatment of $\mathrm{Ph}+$ leukemia in mice. Proceedings of the National Academy of Sciences 103(45): 16870-16875.

15. Pene-Dumitrescu T, Peterson LF, Donato NJ and Smithgall TE (2008) An inhibitor-resistant mutant of Hck protects CML cells against the antiproliferative and apoptotic effects of the broad-spectrum Src family kinase inhibitor A-419259. Oncogene 27(56): 7055-7069.

16. Corey SJ, Dombrosky-Ferlan PM, Zuo S, Krohn E, Donnenberg AD, et al. (1998) Requirement of Src kinase Lyn for induction of DNA synthesis by granulocyte colony-stimulating factor. Journal of Biological Chemistry 273(6): 3230-3235

17. Dai Y, Rahmani M, Corey SJ, Dent P, Grant S (2004) A Bcr/Ablindependent, Lyn-dependent form of imatinib mesylate (STI-571) resistance is associated with altered expression of Bcl-2. Journal of Biological Chemistry 279(33): 34227-34239.

18. Gamas P, Marchetti S, Puissant A, Grosso S, Jacquel A, et al. (2009) Inhibition of imatinib-mediated apoptosis by the caspase-cleaved form of the tyrosine kinase Lyn in chronic myelogenous leukemia cells. Leukemia 23(8): 1500-1506.

19. Warmuth M, Bergmann M, Priess A, Häuslmann K, Emmerich B, et al (1997) The Src family kinase Hck interacts with Bcr-Abl by a kinaseindependent mechanism and phosphorylates the Grb2-binding site of Bcr. Journal of Biological Chemistry 272(52): 33260-33270.

20. Klejman A, Schreiner SJ, Nieborowska SM, Slupianek A, Wilson M, et al. (2002) The Src family kinase Hck couples BCR/ABL to STAT5 activation in myeloid leukemia cells. The EMBO Journal 21(21): 5766-5774.

21. Basmadjian C, Zhao Q, Bentouhami E, Djehal A, Nebigil CG, et al. (2014) Cancer wars: natural products strike back. Frontiers in Chemistry 2: 20.

22. Capdeville R, Buchdunger E, Zimmermann J, Matter A (2002) Glivec (STI571, imatinib), a rationally developed, targeted anticancer drug. Nature reviews Drug discovery 1(7): 493-502.

23. Harries M, Smith I (2002) The development and clinical use of trastuzumab (Herceptin). Endocrine-Related Cancer 9(2): 75-85.

24. Daub H, Specht K, Ullrich A (2004) Strategies to overcome resistance to targeted protein kinase inhibitors. Nature Reviews Drug Discovery 3(12): 1001-1010.

25. Banavath HN, Sharma OP, Kumar MS, Baskaran R (2014) Identification of novel tyrosine kinase inhibitors for drug resistant T315I mutant BCRABL: a virtual screening and molecular dynamics simulations study. Scientific reports 4: 6948 .

26. Parcha P, Sarvagalla S, Madhuri B, Pajaniradje S, Baskaran V, et al. (2017) Identification of natural inhibitors of Bcr-Abl for the treatment of chronic myeloid leukemia. Chemical biology \& drug design 90(4): 596-608.

27. Khajapeer KV, Baskaran R (2016) Natural products for treatment of chronic myeloid leukemia. In: Bankovic J (Ed.), Anti-cancer drugsnature, synthesis and cell. ( $\left.1^{\text {st }} \mathrm{edn}\right)$, Intech publications, Croatia, pp.1-48.

28. Patwardhan B, Gautam M (2005) Botanical immunodrugs: scope and opportunities. Drug Discovery Today 10(7): 495-502.

29. Oaxaca DM, Yang-Reid SA, Ross JA, Rodriguez G, Staniswalis JG, et al. (2016) Sensitivity of imatinib-resistant T315I BCR-ABL CML to a synergistic combination of ponatinib and forskolin treatment. Tumor Biology 37(9): 12643-12654.

30. Chen Y, Zhon Q, Zhang L, Zhong Y, Fan G, et al. (2017) Stellettin B induces apoptosis in human chronic myeloid leukemia cells via targeting PI3K and Stat5. Oncotarget 8(17): 28906-28921.

31. Bonifacio M, Rigo A, Guardalben E, Bergamini C, Cavalieri E, et al. (2012) $\alpha$-bisabolol is an effective proapoptotic agent against BCR-ABL+ cells in synergism with Imatinib and Nilotinib. PloS One 7(10): 46674

32. Bose P, Park H, Al-Khafaji J, Grant S (2013) Strategies to circumvent the T315I gatekeeper mutation in the Bcr-Abl tyrosine kinase. Leukemia Research Reports 2(1): 18-20.

33. Raj L, Ide T, Gurkar AU, Foley M, Schenone M, et al. (2011) Selective 
killing of cancer cells by a small molecule targeting the stress response to ROS. Nature 475(7355): 231-234.

34. Zhang H, Trachootham D, Lu W, Carew J, Giles FJ, et al. (2008) Effective killing of Gleevec-resistant CML cells with T315I mutation by a natural compound PEITC through redox-mediated mechanism. Leukemia 22(6): 1191-1199.

35. Truitt L, Hutchinson C, DeCoteau JF, Geyer CR (2014) Chaetocin antileukemia activity against chronic myelogenous leukemia cells is potentiated by bone marrow stromal factors and overcomes innate imatinib resistance. Oncogenesis 3(10): 122.

36. Chakraborty JB, Mahato SK, Joshi K, Shinde V, Rakshit S, etal. (2012) Hydroxychavicol, a Piper betle leaf component, induces apoptosis of CML cells through mitochondrial reactive oxygen species-dependent JNK and endothelial nitric oxide synthase activation and overrides imatinib resistance. Cancer Science 103(1): 88-99.

37. Sattler M, Verma S, Shrikhande G, Byrne CH, Pride YB, et al. (2000) The (c) (i) Creative Commons Attribution 4.0

(1) International License

For possible submissions Click Here
Submit Article

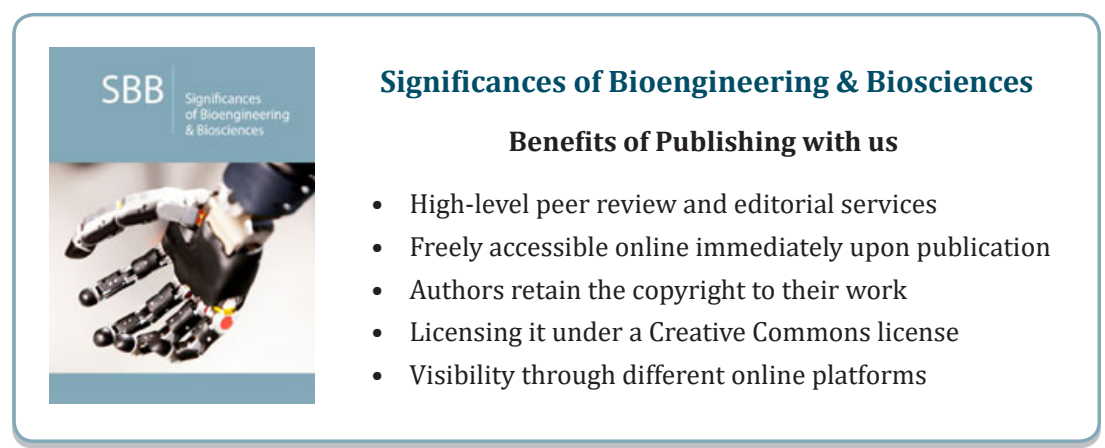

